\title{
Contour-based Hand Pose Recognition for Sign Language Recognition
}

\author{
Mika Hatano, Shinji Sako, Tadashi Kitamura \\ Graduate School of Engineering, Nagoya Institute of Technology \\ \{pia, sako, kitamura\}@mmsp.nitech.ac.jp
}

\begin{abstract}
We are developing a real-time Japanese sign language recognition system that employs abstract hand motions based on three elements familiar to sign language: hand motion, position, and pose. This study considers the method of hand pose recognition using depth images obtained from the Kinect v2 sensor. We apply the contour-based method proposed by Keogh to hand pose recognition. This method recognizes a contour by means of discriminators generated from contours. We conducted experiments on recognizing 23 hand poses from 400 Japanese sign language words.
\end{abstract}

Index Terms: hand pose, contour, sign language recognition, real-time, Kinect

\section{Introduction}

In Japan, Japanese sign language is usually used among hearing impaired people to communicate. In addition, these people often communicate with others through a third person who understands both oral and sign language. The alternative is to use a computer that acts as an interpreter. However, no practical sign language recognition system exists, even one that recognizes isolated words. The difficulties lie in the nature of visual language and its complex structure. Compared with speech recognition, sign language recognition incorporates various visual components, such as hand motions, hand poses and facial expressions. In addition, no established study exists on representing the structure of Japanese sign language in a similar manner to that of spoken language. Therefore, few attempts recognize sign language by units such as hand motions and hand poses [1,2].

Our study develops with real-time recognition of sign language words. In Japanese sign language, a sentence consists of several words and non-manual signals such as facial expressions. To recognize words is a first step and essential to recognize sentences. The number of Japanese sign language words is said to be 3,000 or more. Recognition by discriminators that are independent of every word has proven ineffective. To produce a practical system, analysis and reconstruction of sign language words are critical. We want to emphasize that database of sign language words is required when we analyze such words. However, no established database currently exists for sign language recognition. Therefore, we employ a database from a computerized sign language word dictionary instead.

Our system is based on three elements of sign language: hand motion, position, and pose. This study considers the method of hand pose recognition for our system. Speeding up hand pose recognition is difficult, because of the number and variety of hand poses caused by rotations, altering the angle from the sensor, and diversities in bone structures. This study considers a hand pose recognition using depth images obtained from a single depth sensor. We apply the contour-based method pro- posed by Keogh [3] to hand pose recognition. This method recognizes a contour by means of discriminators learned from contours. We conducted experiments to recognize 23 hand poses from 400 Japanese sign language words.

\section{System overview}

Figure 1 shows the flowchart of the entire system. We use Kinect v2 sensor [4] to obtain data from sign motions produced by an actual person. First, data obtained from the sensor is segmented into sign language words. Second, the three aforementioned elements are recognized individually. Finally, the recognition result is determined by the weighted sum of each score. The recognition process of the hand pose and other two components employs depth data of the hand region and coordinates of joints, respectively. This study partially considers the method of hand pose recognition and does not discuss other processes on the flowchart.

To utilize the structure in sign language recognition requires an expert knowledge of sign language. We apply a database from the computerized sign language word dictionary produced by Kimura [5] to sign language recognition. Our hand pose recognition is based on the classification of hand types employed in this dictionary. Table 1 shows a portion of the database in the dictionary. This database includes approximately 2,600 Japanese sign language words. Each word is represented by specific sign language types in Table 2 and other elements are indicated in Figure 2. For example, the word "red" which belongs to the type 1 in Table 2 is expressed by the dominant hand and the other hand is not used.

\section{Method of hand pose recognition}

Some methods of hand pose estimation classify depth pixels into parts to obtain joint coordinates [6, 7]. However, these methods present difficulties when the palm does not face the

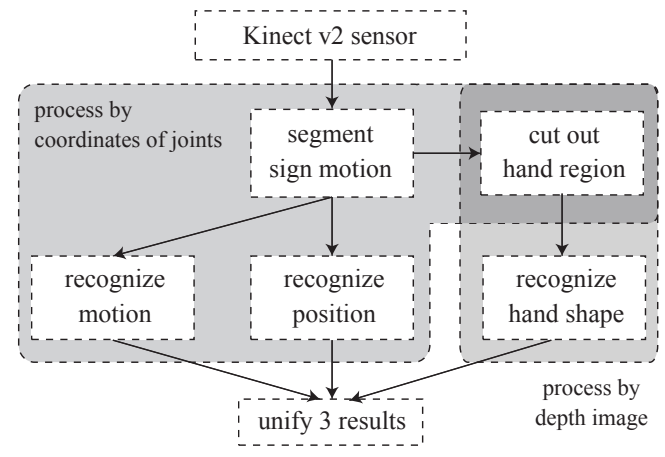

Figure 1: Flowchart of the entire system. 
Table 1: Portion of the database in the dictionary.

\begin{tabular}{|l|c|c|c|c|c|}
\hline Word & $\begin{array}{c}\text { SL } \\
\text { Type }\end{array}$ & $\begin{array}{c}\text { Hand } \\
\text { type }\end{array}$ & $\begin{array}{c}\text { Palm } \\
\text { direction }\end{array}$ & Position & Motion \\
\hline love & 3 & B & down & NS & circle \\
between & 4 & B & side & NS & down \\
blue & 1 & B & back & lower face & back \\
red & 1 & 1 & back & lower face & right \\
baby & 4 & B & up & NS & up-down \\
autumn & 4 & B & back & whole face & front-back \\
open & 4 & B & front & NS & right \\
morning & 1 & S & side & upper face & down \\
shallow & 2 & B & side & NS & up \\
tomorrow & 1 & 1 & front & whole face & front \\
play & 4 & 1 & side & upper face & front-back \\
rain & 4 & 5 & back & NS & up-down \\
walk & 1 & U & back & NS & front \\
relief & 4 & B & back & body & down \\
say & 1 & 1 & side & lower face & front
\end{tabular}

Table 2: Sign Language (SL) types.

\begin{tabular}{|l|c|c|c|c|c|}
\hline & 1 & 2 & 3 & 4 & 5 \\
\hline use both hands & $\times$ & $\bigcirc$ & $\bigcirc$ & $\bigcirc$ & $\bigcirc$ \\
\hline $\begin{array}{c}\text { hand type is same } \\
\text { through two hands }\end{array}$ & & $\bigcirc$ & $\times$ & $\bigcirc$ & $\times$ \\
\hline $\begin{array}{c}\text { non-dominant } \\
\text { hand moves }\end{array}$ & & $\times$ & $\times$ & $\bigcirc$ & $\bigcirc$ \\
\hline
\end{tabular}

camera and some fingers are invisible. We use the contourbased method proposed by Keogh [3]. Contour-based methods work efficiently when recognition objects have distinct shapes. This method treats a contour that encircles an area as a recognition object and uses discriminators called wedges generated from contours. This method is described below.

\subsection{Feature extraction}

Shapes can be converted to distance vectors to form onedimensional series. Figure 3 shows the procedure for extracting a distance vector from a hand image. First, the center point of the hand region is determined by distance transform. Distance transform labels each pixel whose value is " 1 " with the distance to the nearest pixel whose value is " 0 " in a binary image. The center point is a pixel that has a maximal value after distance transform. Next, each distance from the center point to every pixel on the contour is calculated. The distance vector represents a series of these distances.

\subsection{Calculation of distance}

A distance $D$ between two distance vectors $P=$ $\left\{p_{0}, p_{1}, \ldots, p_{n}\right\}$ and $Q=\left\{q_{0}, q_{1}, \ldots, q_{n}\right\}$ is calculated according to the followings.

$$
D(P, Q)=\sqrt{\sum_{i=1}^{n}\left(p_{i}-q_{i}\right)^{2}}
$$

If the length of two distance vectors is different, dynamic time warping (DTW) should be used to adjust for size variations. However, we do not use DTW to avoid excessive computation time. Instead, we unify their length by resizing them in advance.

We can compare contours by calculating their distances or using discriminators generated from contours. These discriminators are called wedges. Wedges have maximal and minimal values at each point. If a contour is located inside a

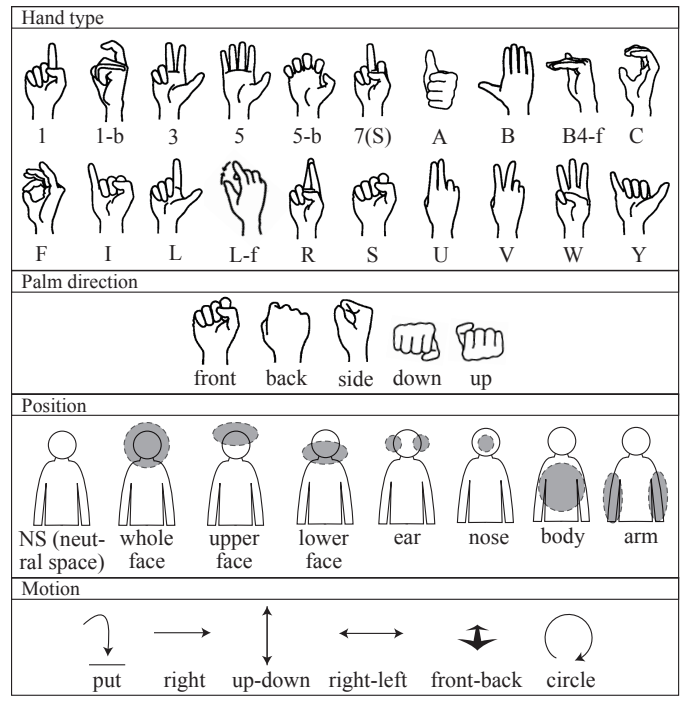

Figure 2: Elements in sign language dictionary.

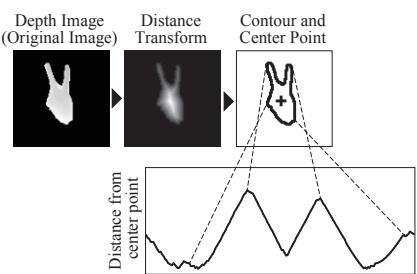

Figure 3: Feature extraction from an image of a hand region.

wedge, the distance is 0 . The distance $D$ between a wedge $W$ ( $U=\left\{u_{0}, u_{1}, \ldots, u_{n}\right\}$ is its top, $L=\left\{l_{0}, l_{1}, \ldots, l_{n}\right\}$ is its bottom) and a contour $P=\left\{p_{0}, p_{1}, \ldots, p_{n}\right\}$ is calculated based on the following equation. For example, the sum of broken lines in Figure 4 is a distance between $W$ and $P$.

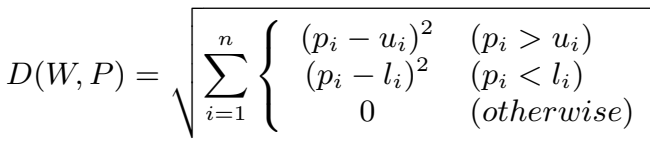

\subsection{Producing wedges}

Wedges are produced according to the following procedures.

1. Extract features from hand images.

2. Calculate distances of all contours.

3. Unify two contours in ascending order of distances. The maximal and minimal values of merged contours become a wedge.

4. Repeat process 3. until the number of wedges decreases to a definite number.

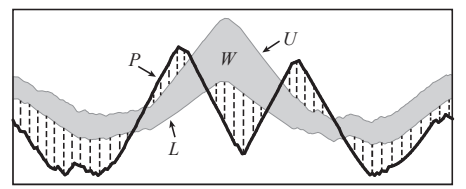

Figure 4: Distance between a wedge and contour. 


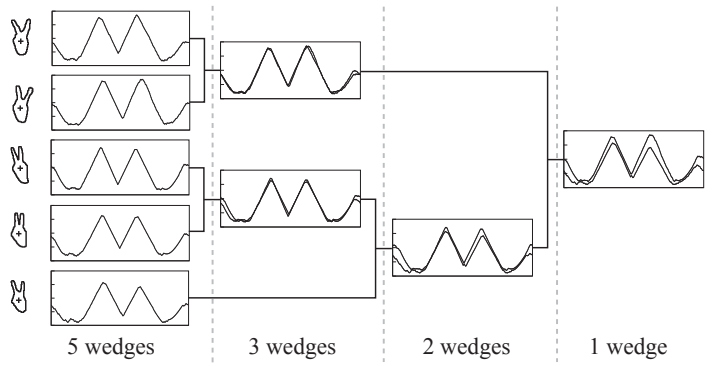

Figure 5: Producing wedges from five contours.

When Figure 5 shows an example of producing wedges. A wide wedge produced by contours that are diverse does not function as a discriminator. We prepare various wedges for recognizing each hand type in order to consider the details of contours.

\subsection{Speeding up calculation}

When we consider a rotation invariant matching of two distance vectors, the calculation must be repeated many times with shifting one of the distance vectors. We can speed up this computation by aborting when the current sum of squared differences exceeds a threshold. In addition, although existing research does not attempt this, we try to speed up the calculation by means of the followings.

- The length of the distance vectors is unified and shortened, and the accuracy does not diminish.

- When the number of wedges per hand type is greater than one, recognition that uses one-by-one wedge is performed prior to help targeting candidates.

\section{Experiments}

\subsection{Datasets}

We conducted experiments on recognizing 23 hand poses in 400 Japanese sign language words in the national sign language test grade 5 . To recognize these 400 words requires to distinguish 23 hand poses in Table 3 defined by hand types and palm directions. Some words have the same hand poses but different position and motion. Our system distinguish each word after recognizing 3 components and unifying recognition results.

Because hand shapes transform with motions, each hand type remains independent even if the palm direction is different. However, some exceptions exist to distinguish sign language words that have the same motion, position, and hand type, but have a different palm direction. For example, Groups 3 and 4 in Table 3 should be distinguished even though the hand type is the same.

To simplify the collection of data in our experiments, we used depth images of stationary hands instead of those obtained during natural sign motions. Table 4 shows the experimental conditions. We conducted four experiments examining the robustness of the recognition method about the variety of hand shapes and the computation time. The objectives of the experiments are described as follows.

Experiment 1 Recognize 100 hand images by wedges produced from the same 100 images per hand type, palm direction, and tester (close-dataset, close-tester).

Experiment 2 Recognize 50 hand images by wedges produced from the other 50 hand images per hand type, palm direc-
Table 3: List of 23 hand pose groups.

\begin{tabular}{|c|c|c|}
\hline ID & Hand type & Palm direction \\
\hline 0 & 1 & front-back, right-left \\
\hline 1 & $1-$ r & right-left \\
\hline 2 & 3 & front-back \\
\hline 3 & 5 & front-back \\
\hline 4 & 5 & up-down \\
\hline 5 & 5 -b & front-back, right-left, up-down \\
\hline 6 & 7 (S) & front-back \\
\hline 7 & A & front-back, right-left \\
\hline 8 & B & front-back \\
\hline 9 & B & right-left \\
\hline 10 & B & up-down \\
\hline 11 & B4-f & right-left \\
\hline 12 & C & right-left \\
\hline 13 & F & front-back \\
\hline 14 & I & front-back \\
\hline 15 & L & front-back \\
\hline 16 & L-f & right-left \\
\hline 17 & R & right-left \\
\hline 18 & S & front-back, right-left, up-down \\
\hline 19 & U & front-back \\
\hline 20 & V & front-back \\
\hline 21 & W & front-back \\
\hline 22 & Y & front-back \\
\hline
\end{tabular}

Table 4: Experimental condition.

\begin{tabular}{|l|l|}
\hline Hand type & 20 types in Figure 2 \\
\hline Palm direction & 3 patterns (front-back, right-left, up-down) \\
\hline Hand pose group & 23 groups* \\
& *determined by hand types and palm directions \\
\hline Tester's profile & A (female, hand size* $16 \mathrm{~cm})$ \\
& B (female, hand size* $18 \mathrm{~cm})$ \\
& C (male, hand size* $19 \mathrm{~cm})$ \\
& D (male, hand size* $21 \mathrm{~cm})$ \\
& $*$ measured from the wrist to the tip of the middle finger \\
\hline Depth image & $100 \times 100$ pixel \\
& 100 images of the hand region \\
& per tester, hand type and palm direction \\
\hline Length of distance vector & 30 or 180 \\
\hline PC specs & OS : Windows 8.164 bit \\
& RAM: 4 GB \\
& CPU : Intel Core i5-4570 $(3.20 \mathrm{GHz}, 4-\mathrm{core})$ \\
\hline
\end{tabular}

tion, and tester (open-dataset, close-tester). Experiments were repeated with different data.

Experiment 3 Recognize 100 hand images of a person by wedges produced from 300 hand images of the other three persons per hand type, and palm direction (opendataset, open-tester). Experiments ware repeated with different data.

Experiment 4 Examine the relationship between the computation time required to recognize a hand image and the average recognition rate from Experiment 2. We attempted to speed up the calculation by the methods in Section 3.4. The threshold value when the calculation was aborted was determined by the preliminary experiment. The length of distance vectors was 30 in this experiment. Each recognition was aided to target candidates as many as five hand pose groups by the recognition that uses one-by-one wedge performed prior.

\subsection{Results}

\subsubsection{Experiment 1, 2}

Figure 6 shows the average recognition rates for Experiment 1 and 2 . The accuracy can be improved by increasing the number of wedges. This can be accomplished because of the variety of hand shapes caused by posing of hand and by altering the angle from the camera. 


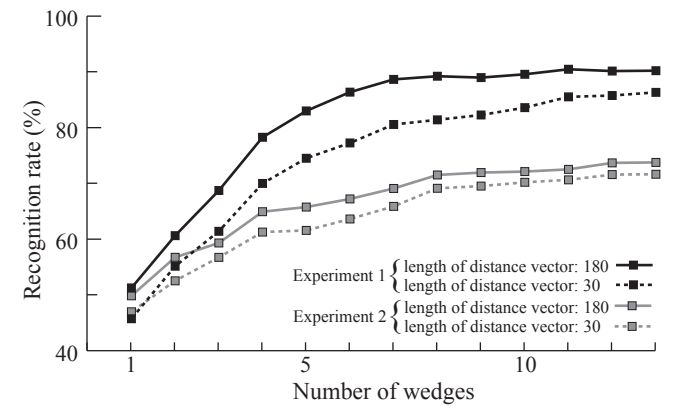

Figure 6: Experiment 1, 2: Recognition rate and number of wedges per hand type and palm direction (person is known).

Experiment 1 was conducted with close-dataset. This experiment is just for sanity check and its condition is impossible in real-life. The result was sufficient for our system. Erroneous recognition in Experiment 1 was primarily caused by misidentifying hand pose Groups 4 and 5. These two groups have a common point that includes a hand pose whose palm direction is down. When we obtain data from a single depth camera, capturing the characteristics of hand shapes when the palm does not face the camera is difficult. Group 6 had the lowest recognition rate among the hand pose group (when the length of the distance vector is 180 , the number of wedges was 10 per hand type and palm direction, and the group's recognition rate was $80 \%$ ). This is because the group was misrecognized as Group 0. These two groups have similar shapes. In addition, the recognition rates of Group 2, 13, 15, 20, and 22 were high under all conditions because other groups do not possess similar shapes.

Experiment 2 was conducted with open-dataset and closetester. The result showed a similar trend to that of Experiment 1 concerning the causes of erroneous recognition. Because no hand shapes from the learning data are included in the evaluation data, the recognition rate was lower than that of Experiment 1. However, no significant difference in recognition rate of Experiment 1 and 2 appeared when the number of wedges is one per hand type and palm direction. Therefore, if the wedges are generated from samples of a certain number, applying unknown data from the same person is possible. The recognition rate from Experiment 2 is expected to approach that of Experiment 1 by increasing the amount of learning data.

Experiments were conducted after changing the length of distance vectors. Although shortening the distance vectors reduces the calculations, the accuracy is expected to fall because of the loss of detailed features. However, no significant differences between the experiments appeared when the length of the distance vectors is 30 and 180 . Therefore, if small sized hand images are used or the contours are rough because of noises, a robust recognition can be accomplished.

The maximal number of wedges was between 20 and 25 in Experiment 1 and between 8 and 13 in Experiment 2. The number fluctuated with the complexity of the hand types.

\subsubsection{Experiment 3}

Experiment 3 was tester-independent setup. Figure 7 shows the results of Experiment 3. The recognition rates shown are the results when the length of distance vectors is 30 . If we change the length to 180 , recognition rates do not change significantly. We specified causes of erroneous recognition when the number of wedges is 30 per hand type and palm direction. The results

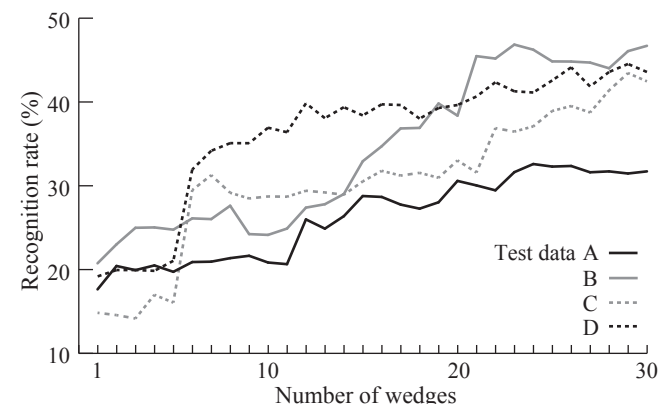

Figure 7: Experiment 3: Recognition rate and number of wedges per hand type and palm direction (person is unknown).

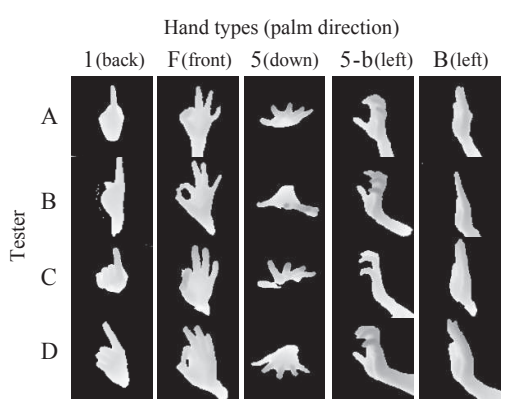

Figure 8: Variety of hand shapes among people.

show the same tendency as in Experiments 1 and 2, that is, $13 \%$ of all data were misrecognized as Groups 4 and 5. The detailed findings for each hand pose group reveal the following: $41 \%$ of Group 6 were misrecognized as Group 0, $53 \%$ of Group 19 were misrecognized as Group 0, $45 \%$ of Group 12 were misrecognized as Group 5.

The low recognition rate is due to individual differences in hand shapes caused by differences in bone structure and posing of hand shown in Figure 8. Wedges produced from the hand images of various people include other hand types. This caused misrecognitions.

Per person details show that the recognition rate was lowest when the system attempted to recognize hand poses of tester A, whose hand size was the smallest. When the number of wedges increases, the recognition rate of tester $\mathrm{B}$, whose hand size is between that of $\mathrm{A}$ and $\mathrm{C}$ is higher than that of other testers.

Although we normalized the scale of distance vectors according to each hand size, hand pose recognition by contours possesses other difficulties when the bone structures are considered. The accuracy diminishes when the system recognizes hand images of a person whose bone structure is dissimilar to any learning data. When we want to recognize hand poses of an unknown person, wedges generated from people who have similar bone structure should be used. Therefore, additional hand images that reveal various characteristics in bone structures should be collected.

\subsubsection{Experiment 4}

Experiment 4 was for checking the computation time. Figure 9 shows the relationship between the computation time required to recognize a hand image and the average recognition rate in Experiment 2. The speed-up process did not affect the recognition rate. 


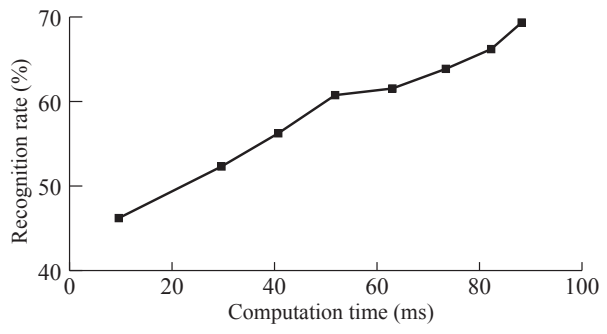

Figure 9: Experiment 4: Average computation time and the recognition rate required to recognize a hand image.

When the person is known, $88 \mathrm{~ms}$ (corresponding to $11 \mathrm{fps}$ ) was required to recognize a hand image with $70 \%$ accuracy. Recognizing all hand images obtained from the sensor with a frame rate of $30 \mathrm{fps}$ is impossible. However, the number of frames required to specify a hand pose is limited because the hand pose does not change at every frame. We can recognize in real-time selected hand images by means of comparison method employing a small calculation such as image moment [8]. This experiment has been implemented in a single-thread. The processing speed can be improved by utilizing a high-speed technique such as multi-threading.

\section{Conclusion}

We are developing a real-time Japanese sign language recognition system based on three elements of sign language: motion, position, and pose. This study examined hand pose recognition by means of contour-based method proposed by Keogh using depth images obtained from a single depth sensor.

We conducted experiments on recognizing 23 hand poses from 400 Japanese sign language words. Under the condition of close-tester, the recognition rate was approximately $90 \%$ for close-dataset, $70 \%$ for open-dataset. In addition, we conducted an experiment to recognize the hand poses of an unknown person by means of discriminators learned from hand poses of other people. The recognition rate dropped considerably because diversities in bone structure of each person's hand generated loose discriminators that are unable to consider the details of contours. We also evaluated the computation time Regarding close-tester and open-dataset, $88 \mathrm{~ms}$ (corresponding to $11 \mathrm{fps}$ ) was required to recognize a hand image with $70 \%$ accuracy.

When we recognize the hand poses of an unknown person, discriminators generated from people who have similar bone structure should be used. Future research in this area requires that hand images of various people be collected and applied for the purpose of recognizing unknown persons.

\section{Acknowledgement}

This research was supported in part by Japan Society for the Promotion of Science KAKENHI (No. 25350666), and Toukai Foundation for Technology.

\section{References}

[1] Rung-Huei Liang and Ming Ouhyoung, "A Real-time Continuous Gesture Recognition System for Sign Language," in Automatic Face and Gesture Recognition, 1998. Proceedings. Third IEEE International Conference on, Apr 1998, pp. 558-567.
[2] Arata Sato and Koichi Shinoda, "Large Vocabrary Sign Language Recognition Based on Cheremes," in IEICE Technical Report PRMU2011-222, SP2011-137, 2012, pp. 155-160.

[3] Eamonn Keogh, Li Wei, Xiaopeng Xi, Sang-Hee Lee and Michail Vlachos, "LB_Keogh Supports Exact Indexing of Shapes under Rotation Invariance with Arbitrary Representations and Distance Measures," in 32nd International Conference on Very Large Data Bases (VLDB2006), 2006, pp. 882-893.

[4] Kinect for Windows, http://kinectforwindows.org

[5] Tsutomu Kimura, Daisuke Hara, Kazuyuki Kanda and Kazunari Morimoto, "Expansion of the System of JSL-Japanese Electronic Dictionary: An Evaluation for the Compound Research System," in Proceedings of the 2nd International Conference on Human Centered Design, ser. HCD'11, 2011, pp. 407-416.

[6] Hui Liang, Junsong Yuan and Daniel Thalmann, "Parsing the Hand in Depth Images," Multimedia, IEEE Transactions on, vol. 16, no. 5, pp. 1241-1253, Aug 2014.

[7] Danhang Tang, Tsz-Ho Yu and Tae-Kyun Kim, "Real-Time Articulated Hand Pose Estimation Using Semi-supervised Transductive Regression Forests," in Proceedings of the 2013 IEEE International Conference on Computer Vision, ser. ICCV '13, 2013, pp. 3224 3231.

[8] Ming-Kuei Hu, "Visual Pattern Recognition by Moment Invariants," Information Theory, IRE Transactions on, vol. 8, no. 2, pp. 179-187, February 1962 